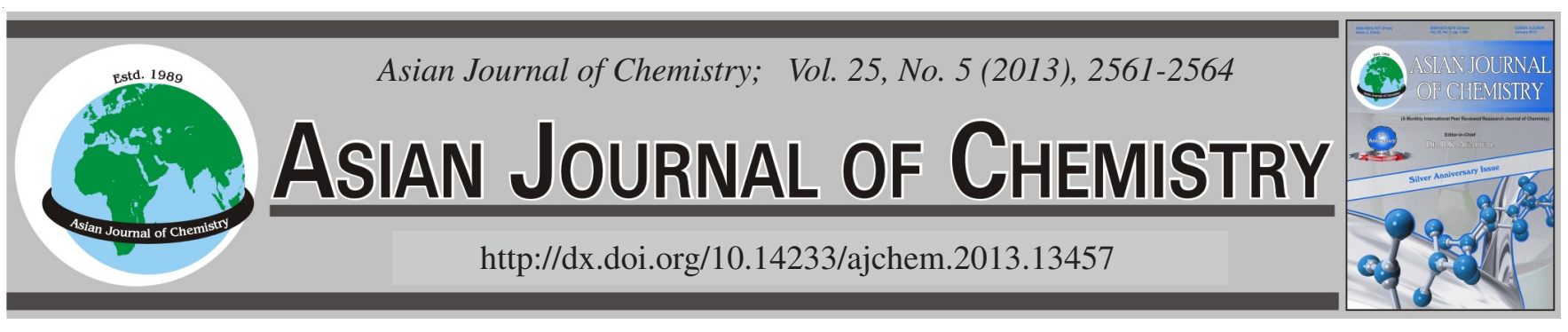

\title{
A Mild and Efficient Procedure for Asymmetric Michael Additions of $\alpha$-Bromochalcone with Cyclohexanone Catalyzed by Different Bases
}

\author{
Li-Guo Gao, Dong-Zhi Liu, Wei Li and Xue-Qin Zhou*
}

School of Chemical Engineering, Tianjin University, Tianjin 300072, P.R. China

*Corresponding author: Fax: +86 22 27400911; Tel: +86 22 27400911; E-mail: zhouxueqin@ tju.edu.cn

\begin{abstract}
A mild and efficient procedure for Michael addition of $\alpha$-bromochalcone with cyclohexanone has been developed. In the presence of sodium ethoxide, $\alpha$-bromochalcone reacted with cyclohexanone to afford Michael products in moderate to high yield and good diastereoselectivities. Especially, while $\mathrm{CH}_{3} \mathrm{CH}_{2} \mathrm{ONa}$ or $t$-BuOK as catalyst, the unexpected products were obtained, which were the compound 4a((8aR,9S)-4a,8a-dihydroxy-10-phenyl-tetradecahydro-phenanthren-9-yl)(phenyl)methanone) and 4b(((8aR,9R)-4a,9dihydroxy-10-phenyl-tetradecahydrophenanthren-9-yl)(phenyl)methanone).
\end{abstract}

Key Words: $\alpha$-Bromochalcone, Cyclohexanone, Synthesis.

\section{INTRODUCTION}

Conjugate addition reactions of $\alpha, \beta$-unsaturated ketones with donors is one of the most important carbon-carbon bondforming reactions in organic synthesis ${ }^{1}$. Over the past decade, there has been attracted increasing interest in the addition reaction of $\alpha, \beta$-unsaturated ketones with cyclohexanone ${ }^{2}$, particularly in Michanel addition reactions. However, most of the reactions used highly activated nucleophiles, such as nitroalkenes ${ }^{3}$ or malonates ${ }^{4}$. Especially, asymmetric Michael addition of enones with ketones is still a challenging reaction. Ishrat ${ }^{5}$ reported the reactions of chalcones with cyclohexanone to generate 1,5-dicarbonyl compounds catalyzed by $\mathrm{CH}_{3} \mathrm{CH}_{2} \mathrm{ONa}$ with good yields (79\%) and low diastereoselectivities which only is ((S)-2-((R)-3-oxo-1,3-diphenylpropyl)cyclohexanone: (R)-2-((R)-3-oxo-1,3-diphenylpropyl)cyclohexanone = $86: 14)$. Many workers ${ }^{6}$ have reported imidazolidinones as organocatalysts to catalyze the reactions of aldehydes with enones, which had some drawbacks such as: long reaction time (more than 4 days) or high catalyst loading and these led to a high cost and limited its application in the pharmaceutical industry. The synthesis of (R)-2-((R)-3-oxo-1,3-diphenylpropyl)cyclohexanone with $\mathrm{CH}_{3} \mathrm{COOLi}$ as catalyst also had the same shortcomings (poor diastereoselectivity). Melchiorre and Jøgensen ${ }^{7}$ have shown that pyrrolidine derivatives could promote the asymmetric conjugate addition smoothly with good results. The reactions were complete in a short time with a low catalyst loading (25\%), but preparation of the organcatalysts was complex and difficult. Therefore, the design and discovery of efficient ways to generate single configuration products is still a challenge in organic synthetic chemistry.

It's noticed that dehydrobromination reaction preceded easily during Michael addition of 1,2,4-triazole to $\alpha$-bromochalcone producing E-1,3-diaryl-3-(1H-1,2,4-triazol-1-yl)2-propen-l-ones in high yields ${ }^{8}$. Considering the reactivity of bromine atom, this paper aims at studying the asymmetric Michael addition of $\alpha$-bromochalcone with cyclohexanone using different catalysts. The compound 1,3-diphenyl-3-(2oxo-cyclohexyl)-1-propanone was obtained with high diastereoselectivity. Besides, the other products was achieved while two mole ratio of cyclohexanones to $\alpha$-bromochalcone in the presence of $t$ - $\mathrm{BuOK}$ or $\mathrm{NaH}$ as catalyst, which has not been reported in the literature.

\section{EXPERIMENTAL}

All the solvents were purified according to standard procedures. The ${ }^{1} \mathrm{H}$ NMR spectra were recorded at $500 \mathrm{MHz}$, ${ }^{13} \mathrm{C}$ NMR spectra were recorded at $500 \mathrm{MHz} .{ }^{1} \mathrm{H}$ and ${ }^{13} \mathrm{C}$ NMR chemical shifts were calibrated to tetramethylsilane as an external reference. Coupling constants are given in hertz. HRMS were recorded on an IonSpec FT-ICR mass spectrometer with ESI resource. Melting points were measured on an RY-I apparatus and uncorrected. The reagents were used without purification. 2-Bromo-1,3-diphenyl-propenone was prepared following literature procedures ${ }^{9}$.

2-(3-Oxo-1,3-diphenyl-propyl)-cyclohexanone $\left(\mathbf{3 a}^{10}\right)$ : Sodium (0.35 g, $0.015 \mathrm{~mol})$ was dissolved in dry ethanol (30 
$\mathrm{mL}$ ) and then cooled to $20{ }^{\circ} \mathrm{C}$, cyclohexanone $(1.96 \mathrm{~g}, 0.02$ $\mathrm{mol})$ and $\alpha$-bromochalcone $(0.98 \mathrm{~g}, 0.01 \mathrm{~mol})$ were added, the mixture was left at same temperature for $3 \mathrm{~h}$. Filtered and the crude solid was washed with ethanol $30 \mathrm{~mL} \times 3 \mathrm{~mL}$, dried, gave a white solid; yield: $80 \%$. (2.74 g, ), m.p. 144.5-145.8 ${ }^{\circ} \mathrm{C}$, IR: 3058, 3025, 2939, 2855, 1709, 1683, 1597, 1495 and $1448 \mathrm{~cm}^{-1},{ }^{1} \mathrm{H}$ NMR $\left(\mathrm{CDCl}_{3}, 500 \mathrm{MHz}\right): \delta(\mathrm{ppm}) 1.25-1.30$ $(\mathrm{m}, 1 \mathrm{H}) 1.55-1.81(\mathrm{~m}, 4 \mathrm{H}), 1.98-2.00(\mathrm{~m}, 1 \mathrm{H}), 2.37-2.43(\mathrm{~m}$, $1 \mathrm{H}), 2.50-2.55(\mathrm{~m}, 1 \mathrm{H}), 2.73\left(\mathrm{dt}, 1 \mathrm{H}, J_{1}=5.5 \mathrm{~Hz}, J_{2}=5.0\right.$ $\mathrm{Hz}), 3.264\left(\mathrm{dd}, 1 \mathrm{H}, J_{1}=10.0 \mathrm{~Hz}, J_{2}=20 \mathrm{~Hz}\right), 3.49(\mathrm{dd}, 1 \mathrm{H}$, $\left.J_{1}=4.0 \mathrm{~Hz}, J_{2}=4.0 \mathrm{~Hz}\right), 3.73\left(\mathrm{dd}, 1 \mathrm{H}, J_{1}=13.5 \mathrm{~Hz}, J_{2}=4.0\right.$ $\mathrm{Hz}), 7.17$ (t, 2H, $J=7.5 \mathrm{~Hz}), 7.258-7.274(\mathrm{~m}, 3 \mathrm{H}), 7.399-$ $7.449(\mathrm{~m}, 2 \mathrm{H}), 7.50(\mathrm{t}, 1 \mathrm{H}, J=7.5 \mathrm{~Hz}), 7.91(\mathrm{~d}, 2 \mathrm{H}, J=8 \mathrm{~Hz})$. HRMS calcd. (\%) for $\mathrm{C}_{21} \mathrm{H}_{22} \mathrm{O}_{2} \mathrm{H}^{+}\left(\mathrm{M}+\mathrm{H}^{+}\right) 307.1693$, found (\%) 307.1686 .

2-(1-Benzyl-2-oxo-2-phenyl-ethyl)-cyclohexanone $\left(\mathbf{3 b}^{\mathbf{1 1}}\right)$ : Catalyst $(0.02 \mathrm{~mol})$ was dissolved in dry solvent $(20 \mathrm{~mL})$, cylohexanone $(1.96 \mathrm{~g}, 0.02 \mathrm{~mol})$ was added, $\alpha$ bromochalcone $(2.08 \mathrm{~g}, 0.01 \mathrm{~mol})$ dissolved in $20 \mathrm{~mL}$ solvent was added, the mixture was kept at same temperature for 12 $\mathrm{h}$, filtered. The crude red solid was purified by column chromatography (petroleum ether:ethyl acetate $=15: 1$ ) to give white solid; yield: $80 \%$. m.p. $144.5-145.8^{\circ} \mathrm{C}$, IR: 3058, 3025, 2939 , 2855, 1709, 1683, 1597, 1495 and $1448 \mathrm{~cm}^{-1},{ }^{1} \mathrm{H} \mathrm{NMR}\left(\mathrm{CDCl}_{3}\right.$, $500 \mathrm{MHz}): \delta$ (ppm) 1.55-1.70 (m, 4H), 1.90-1.91 (m, 1H), 2.00-2.09 (m, 2H), 2.24-2.30 (m, 1H), 2.38-2.41 (m, 1H), 2.70 $\left(\mathrm{dt}, 1 \mathrm{H}, J_{1}=6 \mathrm{~Hz}, J_{2}=5.5 \mathrm{~Hz}\right), 3.40\left(\mathrm{dd}, 1 \mathrm{H}, J_{1}=9.5 \mathrm{~Hz}\right.$,), $3.53\left(\mathrm{dd}, 1 \mathrm{H}, J_{1}=5.0 \mathrm{~Hz}, J_{2}=5.0 \mathrm{~Hz}\right), 3.95\left(\mathrm{dt}, 1 \mathrm{H}, J_{1}=4.5\right.$ $\left.\mathrm{Hz}, J_{2}=5.0 \mathrm{~Hz}\right), 7.149-7.184(\mathrm{~m}, 1 \mathrm{H}), 7.258(\mathrm{t}, 3 \mathrm{H}, J=4.5$ $\mathrm{Hz}), 7.44(\mathrm{t}, 2 \mathrm{H}, J=7.5 \mathrm{~Hz}), 7.53$ (t, 1H, $J=7 \mathrm{~Hz}), 7.96(\mathrm{~d}$, $2 \mathrm{H}, J=7.5 \mathrm{~Hz})$. HRMS calcd $(\%)$ for $\mathrm{C}_{21} \mathrm{H}_{22} \mathrm{O}_{2} \mathrm{H}^{+}\left(\mathrm{M}+\mathrm{Na}^{+}\right)$ 329.1513 , found (\%) 329.1514.

2-[2-(2-Hydroxy-cyclohexylidene)-1-(hydroxy-phenylmethyl)-2-phenyl-ethyl]-cyclohexanone (4a): Catalysts (0.02 $\mathrm{mol})$ dissolved in dry THF $(20 \mathrm{~mL})$, cylohexanone $(1.96 \mathrm{~g}$, $0.02 \mathrm{~mol})$ was added, $\alpha$-bromochalcone $(2.08 \mathrm{~g}, 0.01 \mathrm{~mol})$ dissolved in $20 \mathrm{~mL}$ THF was dropwised in $0.5 \mathrm{~h}$. The mixture was maintained at same temperature for $12 \mathrm{~h}$ and filtered. The crude red solid was purified by column chromatography (petroleum ether:ethyl acetate $=20: 1$ ) to give additional white solid. Yield: $84 \%$. m.p. $184.3-186^{\circ} \mathrm{C}$. IR: $3442.61,3061,3026$, 2930, 2854, 1645, 1595, 1578 and $1493 \mathrm{~cm}^{-1} .{ }^{1} \mathrm{H} \mathrm{NMR}\left(\mathrm{CDCl}_{3}\right.$, $500 \mathrm{MHz}): \delta$ (ppm) 0.99-1.11 (m, 3H), 1.21-1.41 (m, 6H), $1.59(\mathrm{~d}, 1 \mathrm{H}, J=13.5 \mathrm{~Hz}), 1.58-1.86(\mathrm{~m}, 7 \mathrm{H}), 2.07(\mathrm{~d}, 1 \mathrm{H}, J=$ $13.5 \mathrm{~Hz}), 3.26(\mathrm{t}, 1 \mathrm{H}, J=12.0 \mathrm{~Hz}), 3.77(\mathrm{~d}, 1 \mathrm{H}, J=11.5 \mathrm{~Hz})$, $4.78(\mathrm{~s}, 1 \mathrm{H}), 5.18(\mathrm{~s}, 1 \mathrm{H}), 6.89-6.96(\mathrm{~m}, 2 \mathrm{H}), 7.01(\mathrm{dd}, 2 \mathrm{H}$, $J=7.5 \mathrm{~Hz}), 7.19(\mathrm{t}, 1 \mathrm{H}, J=7.5 \mathrm{~Hz}), 7.23(\mathrm{t}, 2 \mathrm{H}, J=7.5 \mathrm{~Hz})$, 7.39 (t, $1 \mathrm{H}, J=7.5 \mathrm{~Hz}), 7.52(\mathrm{~d}, 2 \mathrm{H}, J=7 \mathrm{~Hz}),{ }^{13} \mathrm{C} \mathrm{NMR}(100$ $\left.\mathrm{MHz}, \mathrm{CDCl}_{3}\right): \delta$ (ppm) 19.90, 20.93, 21.30, 24.86, 25.97, 26.17, 36.06, 39.42, 45.59, 50.26, 50.37, 58.96, 72.47, 75.25, $126.02,126.63,128.22,128.35,128.35,128.49,128.49$, 129.06, 131.15, 131.44, 133.39, 140.83, 209.14. HRMS calcd. (\%) for $\mathrm{C}_{27} \mathrm{H}_{32} \mathrm{O}_{3} \mathrm{H}^{+}\left(\mathrm{M}+\mathrm{H}^{+}\right)$405.2424, found (\%) 405.2422 .

2-[2-(2-Hydroxy-cyclohexylidene)-1-(hydroxy-phenylmethyl)-2-phenyl-ethyl]-cyclohexanone (4b): $t$-BuOK $(0.02$ $\mathrm{mol})$ dissolved in dry ethanol $(20 \mathrm{~mL})$, cylohexanone $(1.96 \mathrm{~g}$, $0.02 \mathrm{~mol})$ was added, $\alpha$-bromochalcone $(2.08 \mathrm{~g}, 0.01 \mathrm{~mol})$ dissolved in $20 \mathrm{~mL}$ ethanol was added in $0.5 \mathrm{~h}$, the mixture was left at same temperature for $2 \mathrm{~h}$ under $\mathrm{N}_{2}$ atmosphere, cooled, filtered. The crude red solid was purified by column chromatography (petroleum ether:ethyl acetate $=20: 1$ ) to give additional white solid. Yield: $72 \%$. m.p. $184.5-186.1^{\circ} \mathrm{C}$. IR: 3445, 3060, 3021, 2930, 2854, 1647, 1595, 1578 and 1493 $\mathrm{cm}^{-1} .{ }^{1} \mathrm{H} \mathrm{NMR}\left(\mathrm{CDCl}_{3}, 500 \mathrm{MHz}\right): \delta(\mathrm{ppm}) 1.07-1.57(\mathrm{~m}, 7 \mathrm{H})$ $1.68-1.95(\mathrm{~m}, 4 \mathrm{H}), 2.08(\mathrm{t}, 2 \mathrm{H}, J=12.5 \mathrm{~Hz}), 3.42(\mathrm{t}, 1 \mathrm{H}, J=$ $14 \mathrm{~Hz}), 4.24(\mathrm{~d}, 1 \mathrm{H}, J=14 \mathrm{~Hz}), 5.56(\mathrm{~s}, 1 \mathrm{H}), 6.05(\mathrm{~s}, 1 \mathrm{H})$, 6.89-6.96 (m, 2H), 7.00-7.06 (m, 4H), 7.18-7.23 (m, 4H), 7.28 (s, 2H), $7.43(\mathrm{~d}, 2 \mathrm{H}, J=7 \mathrm{~Hz}),{ }^{13} \mathrm{C}$ NMR $\left(100 \mathrm{MHz}, \mathrm{CDCl}_{3}\right)$ : $\delta$ (ppm) 19.92, 20.91, 21.34, 24.82, 25.98, 26.13, 36.07, 39.44, $45.53,50.25,50.34,58.93,72.44,75.22,126.08,126.61$, $128.27,128.34,128.37,128.41,128.40,129.09,131.11$, $131.40,133.33,140.84,209.10$. HRMS calcd. (\%) for $\mathrm{C}_{27} \mathrm{H}_{32} \mathrm{O}_{3} \mathrm{H}^{+}\left(\mathrm{M}+2 \mathrm{Na}^{+}-\mathrm{H}^{+}\right) 449.2058$, found (\%) 449.2060 .

\section{RESULTS AND DISCUSSION}

Sodium ethoxide was employed to catalyze the conjugated addition of cyclohexanone to $\alpha$-bromochalcone firstly. When the reaction was performed in THF, the product 3a was formed in high diastereoselectivity (>99:1) and low yield (21\%, Table1 , entry 1$)$. When the reaction temperature was increased to $80^{\circ} \mathrm{C}$, the other configuration $(\mathbf{3 b})$ was obtained ( $\left.\mathrm{dr} 100 \%\right)$ in good yield (Table-1, entry 2). While performed in ethanol, high yield was gained with lower diastereoselectivity (94:6, Table-1, entry 3) at room temperature and good results were observed when the temperature was increased to $80{ }^{\circ} \mathrm{C}$ ( $80 \%$ yield, 99:1 diastereoselectivity) (Table-1, entry 4). However, low diastereoselectivities were obtained if toluene was used as solvent either in low temperature or in high temperature (Table-1 entry 5-6). If the temperature was increased to 120 ${ }^{\circ} \mathrm{C}$, the yield of $\mathbf{3}$ nearly decreased to zero (Table- 1 entry 7 ). Then $t$-BuOK was chose as catalyst, which could catalyze the reaction effectively in high yields, but with low diastereoselectivities (Table-1, entry 8-12). Using $\mathrm{NaH}$ as catalyst, the yields of $\mathbf{3}$ were nearly zero at different temperature.

An interesting phenomenon was found. While using $t$-BuOK as the catalyst and the reaction temperature was at 80 ${ }^{\circ} \mathrm{C}$ in ethanol and toluene, then new compounds (4) not 3 were formed (Table-1, entry 13-14) in high yield and mordrate diastereoselectivities. However, when the reaction was carried out under nitrogen atmosphere, only one configuration was obtained (Table-2, entry 1). Changing the catalyst to $\mathrm{NaH}$, only the compound $\mathbf{4 a}$ was afforded in good yield in THF and zero yields of $\mathbf{3}$ or $\mathbf{4}$ in toluene. While changing the feeding order, compounds $\mathbf{3}$ and $\mathbf{4}$ were obtained together as showed in Table-4. Then, the same reactions were done with $\mathbf{3 a}$ and $\mathbf{3 b}$ as starting material to find whether they were the intermediates of the reactions. $4 \mathbf{a}$ was obtained in THF with $\mathrm{NaH}$ as catalyst, but no reaction occurred when $t$-BuOK was used as catalyst in ethanol (Table-4, entry 1).

This fact allowed us to hypothesize that synthesis of compounds $\mathbf{4}$ are preceded as follows: The order also affected the results which could be seen from the Tables 2 and 5 .

From Table-1 (entry 1 and 2), we had believed that $\mathbf{3 b}$ was the thermodynamic control product. But when we did the reactions as showed in Table-3, 3a couldn't convert to $\mathbf{3 b}$ except when THF was used as solvent. This revealed $\mathbf{3 b}$ was not the thermodynamic control product. 


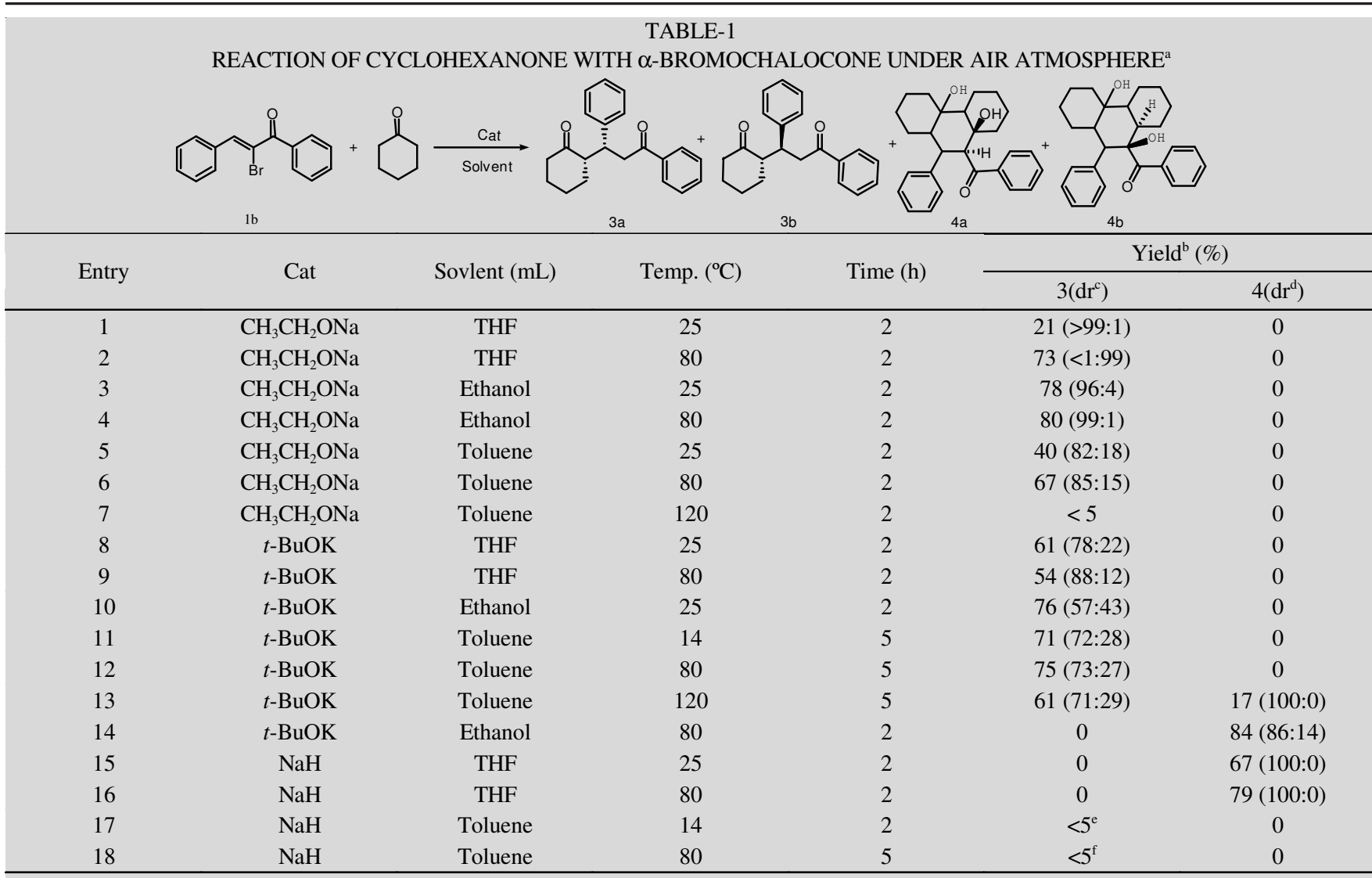

${ }^{a}$ Unless, special remark, the order was mix cyclohexanone and catalyst (in THF) first and stired for five minutes and then added $\alpha$-bromochalcone (in THF) slowly in $0.5 \mathrm{~h}$ in the given temperature. ${ }^{\mathrm{b}}$ Isolated yield. ${ }^{\mathrm{c}} \mathrm{dr}(3 \mathrm{a}: 3 \mathrm{~b})$ was determined by ${ }^{1} \mathrm{H}$ NMR spectroscopy $(500 \mathrm{MHz}) .{ }^{\mathrm{d}}(4 \mathrm{a}: 4 \mathrm{~b}) \mathrm{was}$ determined by ${ }^{1} \mathrm{H}$ NMR spectroscopy $(500 \mathrm{MHz})$. ${ }^{\mathrm{e}} \mathrm{Per}$ cent conversion was $0 .{ }^{\mathrm{f}} \mathrm{Per}$ cent conversion was $100 \%$.

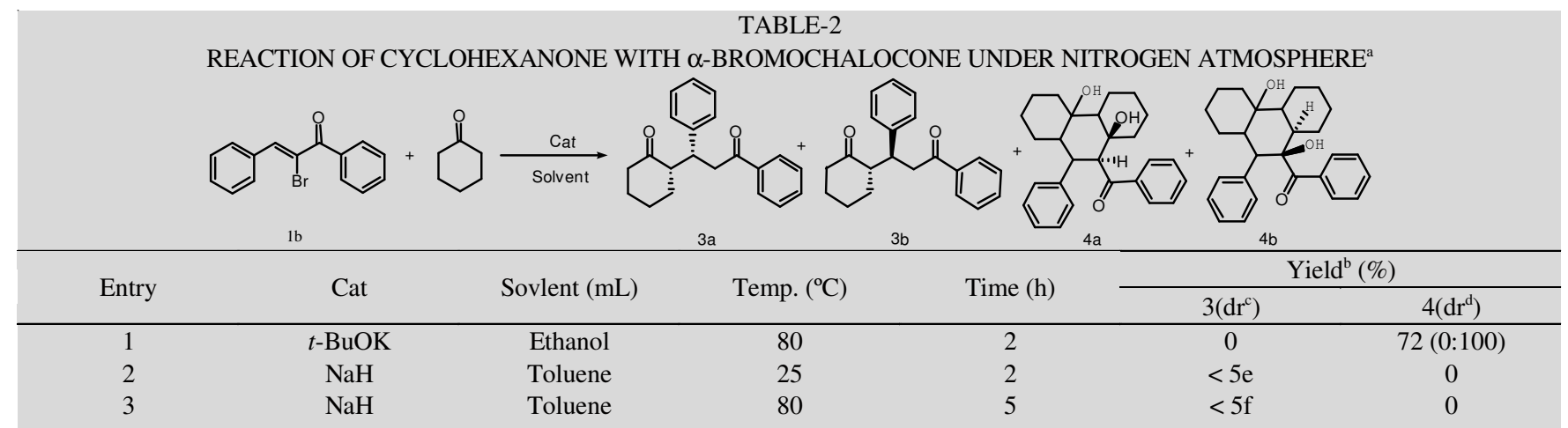

${ }^{a}$ Unless, special remark, the order was mix cyclohexanone and catalyst (in THF) first and stired for five minutes and then added $\alpha$-bromochalcone (in THF) slowly in $0.5 \mathrm{~h}$ in the given temperature. ${ }^{b}$ Isolated yield. ${ }^{\mathrm{c}} \mathrm{dr}(3 \mathrm{a}: 3 \mathrm{~b})$ was determined by ${ }^{\mathrm{l}} \mathrm{H}$ NMR spectroscopy $(500 \mathrm{MHz}){ }^{\mathrm{d}}(4 \mathrm{a}: 4 \mathrm{~b})$ was determined by ${ }^{1} \mathrm{H}$ NMR spectroscopy $(500 \mathrm{MHz})$. ${ }^{\mathrm{e}} \mathrm{Per}$ cent conversion was $0 .{ }^{\mathrm{f}} \mathrm{Per}$ cent conversion was $100 \%$.

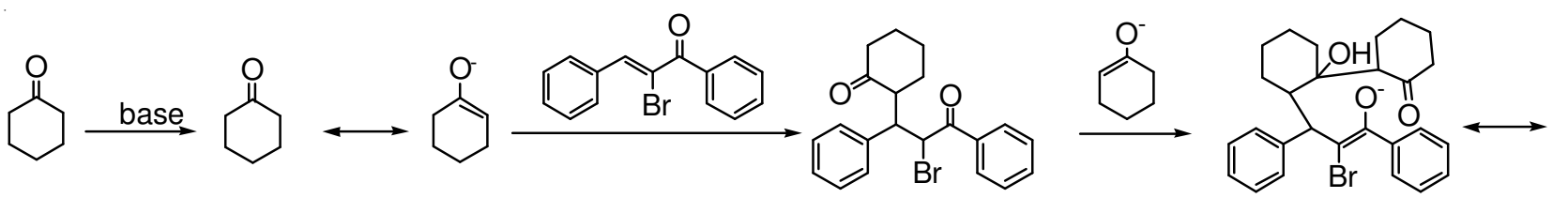

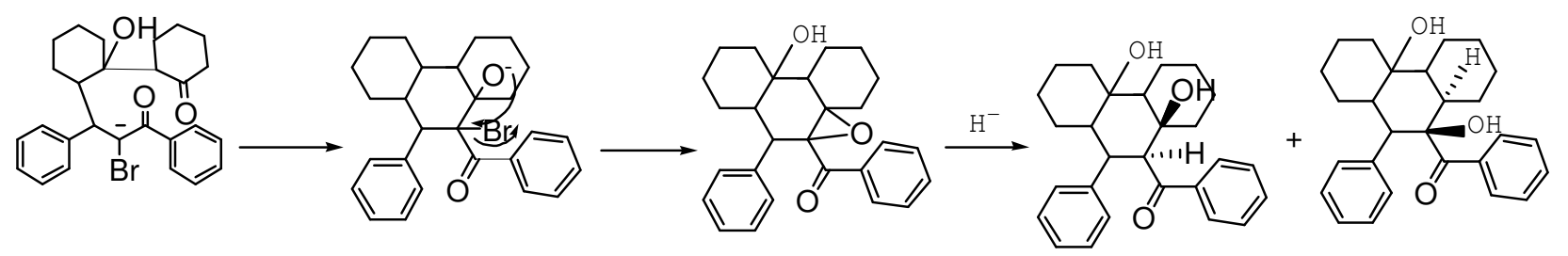

Fig. 1. Proposed mechanism forming compounds 4 
TABLE-4

REACTION OF CYCLOHEXANONE WITH $\alpha$-BROMOCHALOCONE UNDER AIR ATMOSPHERE

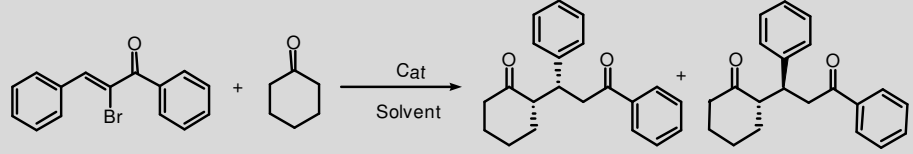

$1 \mathrm{~b}$

$3 a$

$3 \mathrm{~b}$

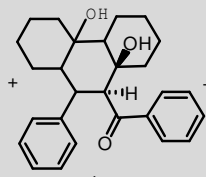

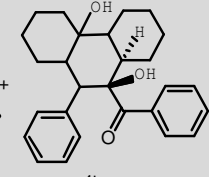

$4 \mathrm{~b}$

\begin{tabular}{|c|c|c|c|c|c|c|}
\hline \multirow{2}{*}{ Entry } & \multirow{2}{*}{ Cat } & \multirow{2}{*}{ Solvent (mL) } & \multirow{2}{*}{ Temp. $\left({ }^{\circ} \mathrm{C}\right)$} & \multirow{2}{*}{ Time (h) } & \multicolumn{2}{|c|}{ Yield $^{\mathrm{b}}(\%)$} \\
\hline & & & & & $3\left(\mathrm{dr}^{\mathrm{c}}\right)$ & $4\left(\mathrm{dr}^{\mathrm{d}}\right)$ \\
\hline 1 & $\mathrm{NaH}$ & THF & 14 & 2 & $57(79: 21)$ & $10(100: 0)$ \\
\hline 2 & $\mathrm{NaH}$ & THF & 25 & 2 & $56(70: 30)$ & $22(100: 0)$ \\
\hline 3 & $\mathrm{NaH}$ & THF & 80 & 2 & $50(79: 21)$ & $27(100: 0)$ \\
\hline
\end{tabular}

${ }^{a}$ Order was mix cyclohexanone and $\alpha$-bromochalcone (in THF) first and stirred for 5 min and then added catalyst (in THF) in the given temperature. ${ }^{b}$ Isolated yield. ${ }^{\mathrm{c}} \mathrm{dr}$ (3a:3b) was determined by ${ }^{1} \mathrm{H}$ NMR spectroscopy $(500 \mathrm{MHz}){ }^{\mathrm{d}}(4 \mathrm{a}: 4 \mathrm{~b})$ was determined by ${ }^{1} \mathrm{H}$ NMR spectroscopy (500MHz).

\begin{tabular}{cccccc}
\multicolumn{7}{c}{ TABLE-3 } \\
\hline \multirow{2}{*}{ Entry } & Cat & $\begin{array}{c}\text { Solvent } \\
(\mathrm{mL})\end{array}$ & $\begin{array}{c}\text { Temp. } \\
\left({ }^{\circ} \mathrm{C}\right)\end{array}$ & $\begin{array}{c}\text { Time } \\
(\mathrm{h})\end{array}$ & $\begin{array}{c}\text { Yield }^{\mathrm{b}} \\
(\%)\end{array}$ \\
\hline 1 & $\mathrm{CH}_{3} \mathrm{CH}_{2} \mathrm{ONa}$ & Ethanol & 80 & 2 & 19 \\
2 & $\mathrm{CH}_{3} \mathrm{CH}_{2} \mathrm{ONa}$ & Toluene & 80 & 2 & 0 \\
3 & $\mathrm{CH}_{3} \mathrm{CH}_{2} \mathrm{ONa}$ & THF & 80 & 2 & 90 \\
\hline
\end{tabular}

${ }^{b}$ Isolated yield.

We found that the addition order of reagents had a great affluence on the products, which were showed in Table-4. The products changed as the order changed when THF was used as solvent.

\begin{tabular}{cccccc} 
& \multicolumn{5}{c}{ TABLE-5 } \\
\cline { 2 - 6 } Entry & Cat & $\begin{array}{c}\text { Solvent } \\
(\mathrm{mL})\end{array}$ & $\begin{array}{c}\text { Temp. } \\
\left({ }^{\circ} \mathrm{C}\right)\end{array}$ & $\begin{array}{c}\text { Time } \\
(\mathrm{h})\end{array}$ & $\begin{array}{c}\text { Yield }^{\mathrm{b}} \\
(\%)\end{array}$ \\
\hline 1 & $\mathrm{NaH}$ & $\mathrm{THF}$ & 80 & 2 & 87 \\
2 & $t$-BuOK & Ethanol & 80 & 2 & 0 \\
3 & $\mathrm{NaH}$ & Toluene & 80 & 5 & 0 \\
\hline
\end{tabular}

\section{Conclusion}

The reaction of $\alpha$-bromochalcone with cyclohexanone generated good results. Different catalysts get different products. Good result was obtained when sodium ethoxide was employed as catalyst in the presence of ethanol. The other configuration was obtained under refluxed when performed in THF with sodium ethoxide as catalyst. Using $t$-BuOK in refluxed ethanol, new compounds 4 were genereted. The single configuration was generated with $\mathrm{NaH}$ as catalyst in THF. At the same time, the feeding order also changed the results of the reaction. Further researches are still in progress.

\section{REFERENCES}

1. P. Perlmutter, Conjugate Addition Reactions in Organic Synthesis, Pergamon: Oxford (1992)

2. (a) G. Wang and H.C. Sun, Catal. Lett., 141, 1324 (2011); (b) H. Gezegen, A. Dingil and M. Ceylan, J. Heterocycl. Chem., 47, 1017 (2010); (c) A. Mastracchio, A.A. Warkentin, A.M. Walji and D.C. MacMillan, Proc. Natl. Acad. Sci. USA, 107, 20648 (2010); (d) Y.O. Sharma and M.S. Degani, Green Chem., 11, 526 (2009).

3. (a) C.M. Yu, J. Qiu, F. Zheng and W.H. Zhong, Tetrahedron Lett., 52, 3298; (b) S. Anwar, H.J. Chang and K. Chen, Org. Lett., 13, 2200 (2011); (c) K. Albertshofer, R. Thayumanavan, N. Utsumi, F. Tanaka and C.F. Barbas, Tetrahedron Lett., 48, 693 (2007); (d) W. Wang, J. Wang and H. Li, Angew. Chem., Int. Ed., 44, 1369 (2005); (e)Y. Hayashi, H. Gotoh, T. Hayashi and M. Shoji, Angew. Chem., Int. Ed., 44, 4212 (2005); (f) T. Ishii, S. Fiujioka, Y. Sekiguchi and H.J. Kotsuki, J. Am. Chem. Soc., 126, 9558 (2004); (g) B.M. Nugent, R.A. Yoder, J.N. Johnson, J. Am. Chem. Soc., 126, 3418 (2004); (h) N. Mase, R. Thayumanavan, F. Tanaka and C.F. Barbas, Org. Lett., 6, 2527 (2004).

4. N. Halland, T. Hansen and K.A. Jørgensen, Angew. Chem. Int. Ed., 42, 4955; (b) N. Halland, P.S. Aburel and K.A. Jørgensen, Angew. Chem. Int. Ed., 42, 661 (2003); (c) S. Hanessian and V. Pham, Org. Lett., 2, 2975 (2000); (d) A. Kawara and T. Taguchi, Tetrahedron Lett., 35, 8805 (1994).

5. R. Ishrat, J. Chem. Soc. Pak., 11, 321 (1989).

6. (a) M.T. Hechavarria Fonseca and B.B. List, Angew. Chem., Int. Ed., 43, 3958 (2004); (b) T.J. Peelen, Y.-G. Chi and S.H. Gellman, J. Am. Chem. Soc., 127, 11598 (2005); (c) Y.-G. Chi and S.H. Gellman, Org. Lett., 7, 4253 (2005).

7. P. Melchiorre and K.A. Jøgensen, J. Org. Chem., 68, 4151 (2003).

8. M.A. Rekhter, G.N. Grushetskaya, A.A. Panasenko and M.Z. Krimer, Chem. Heterocycl. Comp., 31, 792 (1995).

9. (a) G.W. Kabalka, K. Yang, N.K. Reddy and C. Narayana, Synth. Commun., 28, 925 (1998); (b) Ch. Dufraisse, Ann. Chim. Appl., 17, 133 (1922).

10. K. Miura, T. Nakagawa and A. Hosomi, Synlett., 2068 (2003).

11. N. Takashi, F. Hidehiko, N. Yuzo and M. Teruaki, Chem. Lett., 33, 1016 (2004). 